\title{
Pragmatic Recommendations for Identification and Triage of Patients with COVID-19 in Low- and Middle-Income Countries
}

Lia M. Barros, ${ }^{1 \star}$ Jennifer L. Pigoga, ${ }^{2}$ Sopheakmoniroth Chea, ${ }^{3}$ Bhakti Hansoti, ${ }^{4}$ Sarah Hirner, ${ }^{5}$ Alfred Papali, ${ }^{6}$ Kristina E. Rudd, ${ }^{7}$ Marcus J. Schultz, ${ }^{8,9,10}$ and Emilie J. Calvello Hynes ${ }^{11}$ for the COVID-LMIC Task Force and the Mahidol-Oxford Research Unit (MORU)

${ }^{1}$ Division of Cardiology, University of Washington Medical Center, Seattle, Washington; ${ }^{2}$ Division of Emergency Medicine, University of Cape Town, Cape Town, South Africa; ${ }^{3}$ Department of Cardiology, Calmette Hospital, Phnom Penh, Cambodia; ${ }^{4}$ Department of Emergency Medicine, Johns Hopkins, Baltimore, Maryland; ${ }^{5}$ Department of Emergency Medicine, University of Colorado School of Medicine, Denver, Colorado;

${ }^{6}$ Division of Pulmonary and Critical Care Medicine, Atrium Health, Charlotte, North Carolina; ${ }^{7}$ Department of Critical Care Medicine, University of Pittsburgh School of Medicine, Pittsburgh, Pennsylvania; ${ }^{8}$ Mahidol-Oxford Tropical Medicine Research Unit (MORU), Mahidol University, Bangkok, Thailand; ${ }^{9}$ Department of Intensive Care, Laboratory of Experimental Intensive Care and Anesthesiology (L.E.I.C.A), Amsterdam University Medical Centers, Amsterdam, The Netherlands; ${ }^{10}$ Nuffield Department of Medicine, Oxford University, Oxford, United Kingdom; ${ }^{11}$ Department of Emergency Medicine, University of Colorado Hospital, Denver, Colorado

Abstract. Effective identification and prognostication of severe COVID-19 patients presenting to healthcare facilities are essential to reducing morbidity and mortality. Low- and middle-income country (LMIC) facilities often suffer from restrictions in availability of human resources, laboratory testing, medications, and imaging during routine functioning, and such shortages may worsen during times of surge. Low- and middle-income country healthcare providers will need contextually appropriate tools to identify and triage potential COVID-19 patients. We report on a series of LMICappropriate recommendations and suggestions for screening and triage of COVID-19 patients in LMICs, based on a pragmatic, experience-based appraisal of existing literature. We recommend that all patients be screened upon first contact with the healthcare system using a locally approved questionnaire to identify individuals who have suspected or confirmed COVID-19. We suggest that primary screening tools used to identify individuals who have suspected or confirmed COVID-19 include a broad range of signs and symptoms based on standard case definitions of COVID-19 disease. We recommend that screening include endemic febrile illness per routine protocols upon presentation to a healthcare facility. We recommend that, following screening and implementation of appropriate universal source control measures, suspected COVID-19 patients be triaged with a triage tool appropriate for the setting. We recommend a standardized severity score based on the WHO COVID-19 disease definitions be assigned to all suspected and confirmed COVID-19 patients before their disposition from the emergency unit. We suggest against using diagnostic imaging to improve triage of reverse transcriptase (RT)-PCR-confirmed COVID-19 patients, unless a patient has worsening respiratory status. We suggest against the use of point-of-care lung ultrasound to improve triage of RT-PCR-confirmed COVID-19 patients. We suggest the use of diagnostic imaging to improve sensitivity of appropriate triage in suspected COVID-19 patients who are RT-PCR negative but have moderate to severe symptoms and are suspected of a falsenegative RT-PCR with high risk of disease progression. We suggest the use of diagnostic imaging to improve sensitivity of appropriate triage in suspected COVID-19 patients with moderate or severe clinical features who are without access to RT-PCR testing for SARS-CoV-2.

\section{INTRODUCTION}

Effective identification and prognostication of severe COVID19 patients presenting to healthcare facilities are essential to reducing morbidity and mortality associated with SARSCoV-2. The initial algorithms used to screen for and to triage potentially positive COVID-19 patients were largely derived from previous experiences managing severe respiratory illnesses. Unfortunately, it is often impractical to apply these recommendations directly to limited-resource settings in low- and middle-income countries (LMICs). Yet, many of the identification and triage methods applied to the pandemic have been developed in high-income countries (HICs), where critical resources for testing and treatment are abundant. As such, these tools are more likely to incorporate more expensive and time-consuming investigations. Low- and middleincome country facilities often suffer from restrictions in the availability of human resources, laboratory testing, medications, and imaging during routine functioning, and such shortages may worsen during times of surge. To sustain

*Address correspondence to Lia M. Barros, Division of Cardiology, University of Washington Medical Center, 1959 NE Pacific St., Seattle, WA 98195. E-mail: Ibarros@uw.edu healthcare provisions for all patients presenting to their systems during the enduring COVID-19 pandemic, LMIC healthcare providers need contextually appropriate tools to identify and triage potential COVID-19 patients.

\section{METHODS}

An international task force with members from LMICs and HICs, all of whom have direct experience in LMIC settings, developed and appraised a list of key questions surrounding the identification and triage of COVID-19 patients in LMICs. A pragmatic, experience-based appraisal of existing literature was conducted to generate a series of LMIC-appropriate recommendations and suggestions regarding this topic. Online consensus discussions were conducted within the COVID-LMIC Task Force's Triage subgroup to develop a set of clearly defined questions regarding identification and prognostication of COVID-19 patients in LMICS, and the use of diagnostic tools to facilitate these two processes. Following review of questions by the larger task force, the triage subgroup members conducted comprehensive reviews of the relevant literature to identify evidence for recommendations related to each of the posed questions. Literature searches for all questions were conducted through November 4, 2020 in 
four general medical databases (PubMed, Medline, Embase, and Web of Science), the Cochrane Libraries, and Google Scholar. Specific search terminology is noted for each question in the following sections. Reference lists of all included articles were manually screened for additional pertinent literature. It is important to note that the COVID-19 literature base is expanding at an unprecedented pace. All studies through the time of writing were considered for inclusion in this review; however, some were excluded because of their limited relevance to LMICs, and others may be missing if released sometime after completion of the literature review.

Articles were screened at the title, abstract, and full-text level to determine relevance to this review's questions. Those that provided information that possibly posed a solution to a study question were included. To contextualize such data, we classified each piece of evidence as being of high, moderate, low, or very low quality, based largely on the design of the study that generated it. Evidence was then used to derive recommendations, which were ranged as strong or weak, putting emphasis on factors that play a strong role in whether or not these recommendations could be implemented in LMICS, such as safety, availability, and feasibility. Recommendations deemed to be strong were worded as "we recommend ...," and weak recommendations as "we suggest. .."

A full description of the methods is provided in the Appendix.

\section{QUESTIONS}

Three questions regarding the identification and triage of COVID-19 patients in LMICs were formulated by the COVIDLMIC Task Force Triage subgroup:

1. What readily available clinical or diagnostic data, outside of a direct SARS-CoV-2 test, can predict whether a patient is COVID-19 positive in LMICs?

2. What validated triage and severity of illness scoring systems are available to help determine the appropriate level of care for COVID-19 patients in LMICs?

3. Which diagnostic modalities can be used to risk stratify patients with suspected or confirmed COVID-19 in LMICs?

Question 1: What readily available clinical or diagnostic data, outside of a direct SARS-CoV-2 test, can predict whether a patient is COVID-19 positive in LMICs? Rationale. A strategy that can effectively and efficiently prioritize the infectious status of all patients is essential to managing the surge of healthcare needs during the COVID-19 pandemic. Such efforts are known as screening, the process of identifying and isolating patients with COVID-19 risk factors on initial presentation to the healthcare system. Unfortunately, the gold standard for screening-testing all patients, and enforcing isolation and precautions while awaiting results-is impossible even in some of the highest-resourced settings worldwide. Currently, a wide range of screening tools are instead being used to rapidly identify persons under investigation for COVID-19 disease, including those with influenza-like illness. In general, these tools use a combination of clinical and diagnostic data that may include symptoms, ${ }^{1-4}$ vital signs, ${ }^{1-4}$ imaging, ${ }^{1-7}$ and laboratory values. $^{1-4}$ In LMICs, where it is likely unfeasible to conduct laboratory and imaging investigations on an overwhelming portion of patients each day, COVID-19 screening tools that rely on such testing to identify potentially infectious patients are rendered impractical.

A screening tool should be highly sensitive, identifying as many cases as possible to limit risk of exposure for healthcare providers and other patients alike. A pragmatic approach, one that can be conducted with minimal human and physical resources yet still obtains high sensitivity, is indispensable for healthcare facilities as they manage the influx of potentially infectious patients. Identifying such patients will allow for the immediate implementation of universal source control measures, such as isolation, facemasks, eye protection, and other personal protective equipment (PPE), reducing the risk of staff or other patients contracting COVID-19 and further straining the healthcare system. Effective screening tools also allow for a reduction in the use of PPE because interactions between staff and low-risk patients are likely safer. In limited-resource settings, rational allocation of expensive and hard-to-procure PPE is critical to maintaining baseline provision of all health care during the COVID-19 response.

Search results. A search was performed to identify articles as of November 4, 2020 using the following search and/or medical subject headings (MeSH) terms: ("COVID-19" or "SARS-CoV-2" or "2019-nCOV" or "Novel Coronavirus") and ("screening" or "identification").

A total of 61 studies were identified that described COVID19 screening methods outside of direct SARS-CoV-2 testing, 26 of which were from LMICs. Four international guidelines described methods of screening patients for COVID-19 without the use of rapid antigen or reverse transcriptase (RT)-PCR testing. Just three of these tools-one ${ }^{8}$ from the United States and two from China ${ }^{9,10}$ (an upper-middle-income country)included associated validation data.

Evidence. Guidelines from the WHO recommend that all persons undergo a screening of a simple set of questions based on the WHO definition of COVID-19 upon first point of contact with the health system. ${ }^{11}$ To meet the WHO's case definition, a patient must meet a combination of clinical and epidemiological criteria, or present with severe acute respiratory illness. ${ }^{12}$ The $\mathrm{WHO}$ also recommends that communities with high rates of other endemic infections that cause fever (e.g., malaria, dengue, and tuberculosis) also screen for these infections upon presentation per routine protocols. ${ }^{13}$

Conducting a screening process such as WHO's is critical, as recent studies have identified that effective screening can lower nosocomial COVID-19 transmission. ${ }^{14}$ Unfortunately, due to the quickly evolving environment of this pandemic, few studies have even been able to validate the ability of screening tools to successfully identify COVID-19-positive patients: To date, only three studies have provided validation data to support the use of non-SARS-CoV-2 test-based screening methods for potential COVID-19 infection.

Two of these studies were conducted in China. Shi et al. ${ }^{9}$ assessed an internal screening tool that uses respiratory symptoms, fever, and epidemiological history (travel outside of the province or contact with known cases within 14 days) to identify COVID-19-positive patients presenting to the emergency and outpatient departments in Shanghai. Results of this prospective validation found high sensitivity (1) and moderate specificity (0.71), with high negative predictive value (1) and low positive predictive value $(0.18)$. These findings indicate that although the tool is catching all patients who have COVID19 , it is also classifying a substantial number of those without 
COVID-19 as potentially positive. A second Chinese study ${ }^{10}$ used data from patients at multiple fever clinics to develop and validate a screening tool. The tool includes epidemiological history (travel outside of the province or contact with known cases within 14 days), along with age, radiological evidence of pneumonia, and eosinophil and leukocyte levels. Prospective validation of the tool suggests that it has balanced sensitivity and specificity ( 0.83 and 0.78 , respectively), with high negative predictive value $(0.97)$ and moderate positive predictive value (0.32). This study also reported an area under the curve (AUC) of 0.86 , which further supports its validity in accurately identifying COVID-19 cases.

A third study ${ }^{8}$ originating in the United States validated a simplistic, three-step method for identifying COVID-19 cases: Patients were assessed for close contact with a confirmed case, loss of taste or smell, and sore throat to determine potential infectivity. This algorithm was found to be highly sensitive (0.96) at the cost of its specificity (0.53). This low specificity, in addition to a low positive predictive value (0.14), suggests that although nearly all cases are being identified, many noninfectious patients may be classified as infectious in this tool.

After screening and standardized triage according to severity of illness, patients who have been deemed potentially positive for COVID-19 may proceed to secondary screening for further differentiation into suspected, probable, and confirmed cases (positive COVID-19 test). ${ }^{13,15}$ This is particularly important in clinical care locations where testing is unavailable or markedly delayed. Secondary screening tools further separated patients using a combination of repeated initial screening questions and more extensive symptom questionnaires. These surveys often included symptom-based questions (e.g., chills, sore throat, rhinorrhea, congestion, dyspnea, headache, myalgia, arthralgia, diarrhea, and rash), as well as imaging (including chest computerized tomography (CT) ${ }^{1-4}$ lung ultrasound, ${ }^{5,6}$ and chest $X$-ray ${ }^{7}$ ) and laboratory tests (including complete blood count (CBC), ${ }^{3}$ absolute lymphocyte count, ${ }^{1,2}$ inflammatory markers, ${ }^{3}$ and influenza tests ${ }^{4}$ ). SARSCoV-2 rapid and PCR tests were also incorporated into some screening algorithms, presumably because expanding beyond a singular test result can mitigate potential testing inaccuracies and improve sensitivity. To date, no studies have validated secondary screening tools on their ability to accurately identify COVID-19 patients in any setting.

Availability, feasibility, affordability, and safety. The two LMIC-validated COVID-19 screening tools evaluated in this literature review are both based on short algorithms, using easy-to-understand definitions. The brevity and clarity of these tools is crucial, because facility-based screening in LMICs is frequently performed by non-healthcare personnel, such as security guards.

Although two tools were identified with validation data in LMICs, both were set in China and are dependent on some resources. The first tool ${ }^{9}$ mentioned requires that the person conducting screening be able to obtain a temperature, which should be feasible in most LMIC settings. The second tool, ${ }^{10}$ however, relies on two laboratory values and CT scan. The equipment for these investigations may be available in some higher-level facilities in LMICs, but, even in these settings, it is likely infeasible and too expensive to run the high volume of tests that would be needed to screen all potential patients using this algorithm. It is unlikely that the tool offered by $\mathrm{Ma}$ et al. ${ }^{10}$ is pragmatic for use in LMICs. Although the third tool ${ }^{8}$ discussed in relation to this review question was developed for, and validated in, the United States, it was included here because of the limited number of validated tools in LMICs and its feasibility in such settings. It is simplistic in nature, with just three inputs, none of which require anything beyond interview skills. A tool such as this may be ideal in LMICs because it could be implemented at the front door of a healthcare facility and carried out by non-healthcare provider staff.

The high sensitivities and negative predictive values of the three validated tools identified in this review suggest that these tools are arguably safe to use, with most cases being identified and those cases that are negative likely to be true negatives. It must be noted, however, that their validation data may not be generalizable to other settings, especially lower-resourced ones: Although two studies were conducted in the upper-middleincome nation of China, they appear to have been used in wellequipped hospitals. Of concern with all of these tools, however, is their lower than ideal specificities and positive predictive values. It is, indeed, safer to overclassify patients as infectious that are not because the alternative scenario-classifying infectious patients as noninfectious-would increase the risk of nosocomial infection. But, classifying additional patients as positive unnecessarily increases resource utilization in the form of PPE, physical space for isolation, and patient-to-provider ratios. In severely resource-constrained environments, such as those seen in LMICs, this must be balanced against the risks of nosocomial transmission.

Most of these screening tools focused on respiratory symptoms and/or fever, along with epidemiological history. This approach runs the risk of missing the wide range of symptoms associated with COVID-19 infection: As described in the WHO case definition, ${ }^{12}$ a substantial portion of COVID19 cases present atypically and would be missed by these tools. ${ }^{16,17}$ For example, one study of 1,099 COVID-19 cases found that only $43.8 \%$ of patients presented with fever. ${ }^{16}$ Furthermore, it is well documented that patients do a poor job of self-reporting epidemiological history-either by choice or because they do not know of exposures-and the impact of epidemiologic data is limited as community transmission increases. $^{18}$

Considering the higher prevalence of other potentially febrile illness in LMICs, it is important to adhere to WHO's recommendation that these diseases also be screened for upon presentation. Similar to rationale for screening for COVID-19 in all patients, screening for other endemic illnesses also has the potential to improve effective allocation of limited resources such as PPE.

Recommendations and suggestions (Table 1). In LMICs, we recommend that all patients be screened upon first contact with the healthcare system using a locally approved questionnaire to identify individuals who have suspected or confirmed COVID19 (strong recommendation, very low quality of evidence).

In LMICs, we suggest that primary screening tools used to identify individuals who have suspected or confirmed COVID19 include a broad range of signs and symptoms based on standard case definitions of COVID-19 disease (strong recommendation, very low quality of evidence).

In LMICs, we recommend that screening include endemic febrile illness per routine protocols upon presentation to a healthcare facility (weak recommendation, low quality of evidence). 
Question 2: What validated triage severity of illness scoring systems are available to help determine appropriate level of care for COVID-19 patients in LMICs? Rationale. The clinical management of patients with COVID-19 can be informed by three key information-gathering and assessment steps: screening, triage, and severity scoring. Screening, discussed previously, serves primarily as a mechanism of isolating patients who are potentially positive cases of COVID-19 to reduce the spread of the virus within facilities. Triage is a systematic method of sorting patients by the severity of their clinical syndrome and matching patients with healthcare resources, regardless of their specific disease state. Severity scoring can then be used to prognosticate patients, although it is applied to a specific disease state, such as confirmed or suspected COVID-19; it is used to risk stratify patients and identify those likely to have poor outcomes such as admission to an intensive care unit or high dependency unit, or death. Like triage, severity scoring can aid providers in targeting limited resources toward those in greatest need.

The WHO has described the severity of COVID-19 cases as mild, moderate, severe, and critical. ${ }^{13}$ Mild patients have upper respiratory tract disease, moderate patients have pneumonia but do not require oxygen, and severe patients require oxygen, and critical patients have acute respiratory distress syndrome or sepsis/septic shock and often require ventilation. Multiple studies have described laboratory and imaging findings, symptoms, and comorbidities that are linked to the severity of COVID-19. ${ }^{19,20}$ Clear guidance on how severity indicators can be used to guide clinical management would enable early recognition and more targeted treatment of the most at-risk patients, improving outcomes even when resources are scarce.

Search results. A search was performed to identify articles as of November 4, 2020 using the following search and/or MeSH terms: ("COVID-19" or "SARS-CoV-2" or "2019-nCOV" or "Novel Coronavirus") and ("triage" or "risk" or "severity" or "stratification" or "prediction" or "index" or "score").

A total of 25 studies were identified that described triage protocols for COVID-19 patients, 11 of which were from LMICs. There were only two ${ }^{21}$ validation studies related to these tools, one of which was from China, ${ }^{22}$ an uppermiddle-income country. There were 48 severity scoring tools described in the literature, 23 of which were from LMICs. Thirty-four of these severity scoring tools included associated validation data, including 17 LMIC validations (15 from China $^{23-36}$ and two from Turkey). ${ }^{37,38}$

Evidence. This review identified a limited number of validated triage tools and a more robust number of validated severity scoring tools.

To date, just two triage tools have published validation data. Only one of these, from China, is mentioned here. A second tool, from the United States, was found to be less sensitive and specific, despite including a greater number of resourceintensive inputs. $^{21}$

In the Jiangsu Province of China, Sun et al. ${ }^{22}$ validated a homegrown system for triaging confirmed COVID-19 patients. The algorithm includes seven inputs: age, blood oxygen saturation, CT scan results, heart rate, lymphocyte count, oxygenation requirements, and respiratory rate. Retrospective validation identified that the tool has high sensitivity (0.955) and specificity (0.899), and an AUC of 0.962 .

In comparison to screening and triage tools, a much larger number of severity scoring tools (34) provided validation data, both in HICs and LMICs. Highlighted here are those most likely to be feasible in LMICs, based on assumptions about facility capacity as described by the Disease Control Priorities, Third Edition. ${ }^{39}$ In this context, only five LMIC-validated scoring systems are considered readily feasible; all of these originate in China and are tools that existed for broader prognostication purposes before the COVID-19 pandemic.

A large review of seven previously developed scoring systems for pneumonia and sepsis was conducted by Fan et al. ${ }^{35}$ This study, which evaluated the capability of tools to predict in-hospital mortality, identified that two tools with feasibility in LMICs - the CRB-65 and quick sequential organ failure assessment (qSOFA)-were predictive of severe COVID-19 illness, with AUC scores of 0.80 and 0.73 , respectively. The CRB-65 tool uses Glasgow Coma Scale (GCS), respiration rate, age older than 65 years, and blood pressure (diastolic or systolic) as inputs, and qSOFA uses GCS, respiratory rate, and systolic blood pressure. Although the study also found other tools to be predictive, all of those not mentioned here relied on resource-intensive laboratory and imaging investigations.

Peng et al. ${ }^{33}$ evaluated the use of the National Early Warning System (NEWS), a previously developed tool, in the context of COVID-19 patients, both with and without the inclusion of age. The traditional NEWS tool includes body temperature, heart rate, level of consciousness, oxygen saturation, respiratory rate, and systolic blood pressure. This study found AUC scores of 0.837 for NEWS and 0.846 for NEWS and age when the tool is used with confirmed COVID-19 patients. National Early Warning System had a sensitivity of 0.917 and specificity of 0.574 , whereas NEWS and age had a slightly higher sensitivity of 1.0 and lower specificity of 0.511 .

Hu et al., ${ }^{36}$ evaluated two previously available rapid scoring systems as predictors of in-hospital mortality of 138 COVID19 patients in China. The Modified Early Warning Score predicted in-hospital mortality with a sensitivity and specificity of $68.4 \%$ and $65.1 \%$, respectively. This scoring tool includes body temperature, GCS, heart rate, respiratory rate, and systolic blood pressure. The Rapid Emergency Medicine Score (REMS) predicted in-hospital mortality with a sensitivity and specificity of 0.8947 and 0.6977 , respectively. The REMS model uses age, GCS, heart rate, mean arterial pressure, and respiratory rate to predict mortality. ${ }^{36}$ Notably, these scoring systems primarily differ by the inclusion of oxygen saturation and patient age in REMS, which has a higher sensitivity for COVID severity screening. Including oxygen saturation in severity scoring tools may therefore be useful for maximizing sensitivity. Directing efforts toward procuring and maintaining working blood oxygen saturation monitoring equipment may be an effective means of improving triage accuracy; however, it is essential that tools be made available with and without this input, as blood oxygen saturation monitoring equipment may remain limited in availability in LMICs. ${ }^{39,40}$

Availability, feasibility, affordability, and safety. Both triage and scoring tools that are based on laboratory studies and imaging may have limited utility in low-resource settings that lack access to advanced testing. Fortunately, multiple severity scoring algorithms have been validated in LMICs; however, as has been mentioned previously for screening and triage tools, these tools' generalizability outside of upper-middle-income nations may be very limited. ${ }^{41}$ This review found only one triage system, providing limited evidence on what tool inputs may be effective in triaging COVID-19 patients in LMICs. The 
TABLE 1

Recommendations and suggestions on screening, triage, and severity scoring in COVID-19 patients in LMICs

1. Screening tools

2. Triage severity of illness scoring tools

3. Stratification through diagnostic imaging
1.1. In LMICs, we recommend that all patients be screened upon first contact with the healthcare system using a locally approved questionnaire to identify individuals who have suspected or confirmed COVID-19 (strong recommendation, very low quality of evidence).

1.2. In LMICs, we suggest that primary screening tools used to identify individuals who have suspected or confirmed COVID-19 include a broad range of signs and symptoms based on standard case definitions of COVID-19 disease (strong recommendation, very low quality of evidence).

1.3. In LMICs, we recommend that screening include endemic febrile illness per routine protocols upon presentation to a healthcare facility (weak recommendation, low quality of evidence).

2.1. In LMICs, we recommend that, following screening and implementation of appropriate universal source control measures, suspected COVID-19 patients be triaged with a triage tool appropriate for the setting (strong recommendation, very low quality of evidence).

2.2. In LMICs, we recommend a standardized severity score based on the WHO COVID-19 disease definitions be assigned to all suspected and confirmed COVID-19 patients before their disposition from the emergency unit (weak recommendation, low quality of evidence).

3.1. In LMICs, we suggest against using diagnostic imaging to improve triage of RT-PCR-confirmed COVID-19 patients, unless a patient has worsening respiratory status (weak recommendation, very low quality of evidence).

3.2. In LMICs, we suggest against the use of point-of-care lung ultrasound to improve triage of RTPCR-confirmed COVID-19 patients (weak recommendation, low quality of evidence).

3.3. In LMICs, we suggest the use of diagnostic imaging to improve sensitivity of appropriate triage in patients who are RT-PCR negative but have moderate-to-severe symptoms and concern for a false-negative RT-PCR and with a high risk of disease progression (weak recommendation, very low quality of evidence).

3.4. In LMICs, we suggest the use of diagnostic imaging to improve sensitivity of appropriate triage in suspected COVID-19 patients with moderate or severe clinical features who are without access to RT-PCR testing for SARS-CoV-2 (weak recommendation, very low quality of evidence).

LMICs = low- and middle-income countries; RT-PCR = reverse transcriptase-PCR. Grading: see Appendix for explanations.

other validated triage tool identified in this search was from a $\mathrm{HIC}$, and its extensive laboratory and imaging requirements likely render it impractical in many resource settings; it therefore cannot be recommended for use.

Recommendations and suggestions (Table 1). In LMICs, we recommend that, following screening and implementation of appropriate universal source control measures, suspected COVID-19 patients be triaged with a triage tool appropriate for the setting (strong recommendation, very low quality of evidence).

In LMICs, we recommend a standardized severity score based on the WHO COVID-19 disease definitions be assigned to all suspected and confirmed COVID-19 patients before their disposition from the emergency unit (weak recommendation, low-quality evidence).

Question 3: Which diagnostic modalities can be used to risk stratify patients with suspected or confirmed COVID19 in LMICs? Rationale. In addition to the history and examination, HICs frequently include diagnostic modalities such as chest CT, lung ultrasound, and chest X-ray to support a clinical diagnosis of COVID-19. It is not always feasible for these tests to be performed routinely for every patient presenting to acute healthcare systems in LMICs. ${ }^{42}$ High costs to both the patient and health system, insufficient material resources (e.g., lack of ultrasound or CT machines), and limited human resources (e.g., lack of training in use of point-of-care ultrasound or inadequate numbers of healthcare providers to perform these tests) make it challenging to use these diagnostic modalities routinely for triage. However, when available, targeted use of diagnostic modalities can increase the likelihood of early diagnosis, especially when testing resources are limited or unavailable. Please refer to the separate article within this Journal issue on testing and diagnosis for further discussion of diagnostic modalities, including laboratory diagnostics (reference).

Search results. A search was performed to identify articles as of November 4, 2020 using the following search and/or
MeSH terms: ("COVID-19" or "SARS-CoV-2" or "2019-nCOV" or "Novel Coronavirus") and ("ultrasound" or "CT" or "computerized tomography" or "radio" or "X-ray" or "imag" or "oximet") and ("screening" or "sensitivity" or "diagnosis" or "triage" or "identif*" or "severity").

The literature search revealed 32 results. Of these, most were narrative reviews or qualitatively described the clinical experience at a single center. None specifically evaluated the utility of diagnostic modalities in enhancing the sensitivity of triage protocols.

Evidence. Some small, retrospective studies have found chest CT scans to have greater sensitivity but far lower specificity than RT-PCR testing in identifying symptomatic patients positive for COVID-19 disease (i.e., positive follow-up RT-PCR test). ${ }^{26,27}$ In a single-center, retrospective, observational study from China, of 15 patients who were ultimately found to have COVID-19 disease on serial RT-PCR testing, but initially having had a negative RT-PCR result, $66.7 \%$ had chest CT imaging findings consistent with COVID-19 before the initial negative RT-PCR test, and $93 \%$ had chest CT findings consistent with COVID-19 before or in parallel to receiving a positive follow-up RT-PCR test. ${ }^{26}$ Similarly, another Chinese single-center retrospective study including 36 patients who were ultimately found to have COVID-19 disease reported that abnormal chest CT on presentation was $97.2 \%$ sensitive (35 of 36 patients) for COVID-19, whereas initial RT-PCR was only $83 \%$ sensitive (30 of 36 patients). ${ }^{43}$ To support the use of noncontrast chest CT as a predictor for lung involvement of COVID-19, the Dutch Radiological Society published a framework, the COVID-19 Reporting and Data Systems (CORADS). This study found a very good performance for predicting COVID-19 in patients with moderate-to-severe symptoms and has substantial interobserver agreement, especially for patients categorized to low or high suspicion, but from a HIC and may have limited applicability in LMICs. ${ }^{44}$ 
Although abnormalities on chest radiographs are common in symptomatic patients found to have COVID-19 disease, these have a lower sensitivity than initial RT-PCR, ${ }^{45}$ and also have a lower sensitivity than chest CT scans. ${ }^{43}$ In a small, multicenter, retrospective, observational study including patients who would ultimately test positive for COVID-19, baseline chest radiograph had a sensitivity of $69 \%$, compared with $91 \%$ for initial RT-PCR. Chest radiograph abnormalities preceded a positive RT-PCR in only six of 64 patients $(9 \%) .{ }^{44}$ Given this body of evidence, the American College of Radiology has recommended against chest imaging as a screening modality, even in high-resource settings where such imaging might be readily available. ${ }^{46}$

Point-of-care lung ultrasound has been proposed as a sensitive and relatively inexpensive diagnostic modality to augment laboratory-based diagnosis and triage protocols. ${ }^{47}$ Small observational studies and experiential reports have demonstrated high sensitivity of lung ultrasound to detect COVID-19 disease and utility in reducing use of other imaging modalities, ${ }^{47,48}$ and multiple groups have investigated integrating ultrasound in the screening and triage process. ${ }^{5,6,49-52}$ However, none of these studies were set in LMICs, and we did not identify studies showing additional benefit from ultrasound in the triage process beyond standard clinical and laboratory-based approaches. Therefore, we do not currently recommend such a practice be used in LMICs.

The Fleischner Society, an international, multidisciplinary medical society for thoracic radiology, has published a consensus statement on the role of chest imaging in the management of confirmed or suspected COVID-19 patients. ${ }^{53}$ Drafted by experts from $\mathrm{HIC}$, the recommendations in this document consider severity of disease (mild versus moderate-to-severe) as well as the environment of care (resource-constrained versus non). Although no experts from LMICs were represented in this committee, in resource-constrained environments, the document advises the use of chest imaging in patients with moderate-to-severe features on COVID-19 "to support more rapid triage of patients when point-ofcare COVID-19 testing is not available or results are negative." Additional groups have recommended a similar approach, combining chest CT with RT-PCR to improve sensitivity of initial evaluation for patients in whom there is a high clinical suspicion for COVID-19. ${ }^{54}$

Availability, feasibility, affordability, and safety. Availability of radiologic diagnostic modalities to augment triage will be impacted by material resources (e.g., whether the machines are available at a given center), status of the machines (e.g., ability to repair and maintain them, and ability to properly clean them after use on potentially infectious patients), and availability of clinical staff who are skilled in performing and interpreting the studies. Although each of these elements will be largely influenced by the resources available before the COVID-19 pandemic, it is likely that they will potentially be negatively impacted in the setting of the pandemic. This means that imaging modalities that may usually be available at a center may be temporarily unavailable (or less available) during the COVID-19 pandemic because of human and material resource strains.

Feasibility of these studies will likewise largely be determined by the pre-pandemic resources of individual clinical sites, as increasing capacity to perform diagnostic imaging in the setting of the pandemic will be challenging.

Affordability must be considered in the context of affordability to the healthcare system and affordability to the patient. Some imaging modalities, such as CT and radiographs, are more likely to be billed directly to the patient or their insurer, whereas modalities such as point-of-care lung ultrasound may be more "affordable" to the patient if they are not billed for. However, point-of-care ultrasound may require time from the primary bedside clinician, whereas radiographs and CT scans typically are performed by ancillary teams. In situations where the providers performing initial triage have increased clinical load (such as during a pandemic), it may be less feasible to perform point-of-care ultrasound because of limited time. In addition, in resource-limited settings, point-ofcare lung ultrasound is less frequently performed without appropriate quality assurance programs and skill building programs, also challenging feasibility. ${ }^{55}$

Although radiation exposure is always a safety consideration, this exposure is relatively benign when considering the use of one or a few additional imaging tests per patient. However, transportation of potentially severely ill patients to radiology departments to perform radiographs or CT scans introduces additional risk of clinical decompensation. In addition, use of radiologic equipment (e.g., CT or ultrasound machines) or spaces (e.g., imaging suites) for highly infectious patients introduces the risk of viral transmission to other patients or staff. This risk, and the time and resources required to mitigate it through cleaning, must be considered.

Recommendations and suggestions (Table 1). In LMICs, we suggest against using diagnostic imaging to improve triage of RT-PCR-confirmed COVID-19 patients, unless a patient has worsening respiratory status (weak recommendation, very low quality of evidence).

In LMICs, we suggest against the use of point-of-care lung ultrasound to improve triage of RT-PCR-confirmed COVID-19 patients (weak recommendation, low quality of evidence).

In LMICs, we suggest the use of diagnostic imaging to improve sensitivity of appropriate triage in patients who are RT-PCR negative but have moderate to severe clinical features and concern for a false-negative RT-PCR and with a high risk of disease progression (weak recommendation, very low quality of evidence).

In LMICs, we suggest the use of diagnostic imaging to improve sensitivity of appropriate triage in suspected COVID-19 patients with moderate or severe clinical features who are without access to RT-PCR testing for SARS-CoV-2 (weak recommendation, very low quality of evidence).

Received August 22, 2020. Accepted for publication December 20, 2020.

Published online January 6, 2021.

Note: The Appendix can be found at www.ajtmh.org.

Financial support: Open access fees for this article and all others in this issue were supported by the Wellcome Trust of Great Britain.

Authors' addresses: Lia M. Barros, Division of Cardiology, University of Washington Medical Center, Seattle, WA, E-mail: Ibarros@uw.edu. Jennifer L. Pigoga, Division of Emergency Medicine, University of Cape Town, Cape Town, South Africa, E-mail: jennpigoga@ gmail.com. Sopheakmoniroth Chea, Department of Cardiology, Calmette Hospital, Phnom Penh, Cambodia, E-mail: nirothsopheak@ gmail.com. Bhakti Hansoti, Department of Emergency Medicine, Johns Hopkins, Baltimore, MD, E-mail: bhansot1@jhmi.edu. Sarah Hirner, Department of Emergency Medicine, University of Colorado School of Medicine, Denver, CO, E-mail: sarah.hirner@cuanschutz. edu. Alfred Papali, Division of Pulmonary and Critical Care Medicine, Atrium Health, Charlotte, NC, E-mail: alfred.papali@atriumhealth.org. Kristina E. Rudd, Department of Critical Care Medicine, University of Pittsburgh School of Medicine, Pittsburgh, PA, E-mail: ruddk@ pitt.edu. Marcus J. Schultz, Mahidol-Oxford Tropical Medicine Research Unit (MORU), Mahidol University, Bangkok, Thailand, Department of Intensive Care, and Laboratory of Experimental Intensive 
Care and Anesthesiology (L.E.I.C.A), Amsterdam University Medical Centers, Amsterdam, The Netherlands, and Nuffield Department of Medicine, Oxford University, Oxford, United Kingdom, E-mail: marcus.j.schultz@gmail.com. Emilie J. Calvello Hynes, Department of Emergency Medicine, University of Colorado Hospital, Denver, CO, E-mail: emilie.calvellohynes@cuanschutz.edu.

This is an open-access article distributed under the terms of the Creative Commons Attribution (CC-BY) License, which permits unrestricted use, distribution, and reproduction in any medium, provided the original author and source are credited.

\section{GROUP MEMBERS OF THE "COVID-LMIC TASK FORCE”}

Heads: Alfred Papali (Atrium Health, Charlotte, NC) and Marcus Schultz (Mahidol University, Bangkok, Thailand; University of Oxford, Oxford, United Kingdom; Amsterdam University Medical Centers, location "AMC," Amsterdam, The Netherlands).

Advisors: Neill K. J. Adhikari (Interdepartmental Division of Critical Care Medicine, University of Toronto, Toronto, Canada); Emilie J. Calvello Hynes (University of Colorado School of Medicine, Department of Emergency Medicine, Denver, CO); Martin Dünser (Kepler University Hospital and Johannes Kepler University Linz, Linz, Austria) and Mervyn Mer (University of the Witwatersrand, Johannesburg, South Africa).

Subgroup members, in alphabetical order: Andrew Achilleos (Sunnybrook Health Sciences Centre, Toronto, Canada); Hanan Y. Ahmed (Department of Internal Medicine, College of Health Sciences, Addis Ababa University, Addis Ababa, Ethiopia). Kevan Akrami (Universidade Federal da Bahia, Salvador, Brazil and University of California San Diego, CA); Lia M. Barros (University of Washington, Seattle, WA); B. Jason Brotherton (AIC Kijabe Hospital, Kijabe, Kenya); Sopheakmoniroth Chea (Calmette Hospital, Phnom Penh, Cambodia); William Checkley (The Johns Hopkins University School of Medicine and The Johns Hopkins University Center for Global Health, Baltimore, MD); Elif Cizmeci (Interdepartmental Division of Critical Care Medicine, Sunnybrook Health Sciences Centre, University of Toronto, Toronto, Canada); Natalie Cobb (University of Washington, Seattle, WA); Finot Debebe (Department of Emergency Medicine and Critical Care, Addis Ababa University School of Medicine, Addis Ababa, Ethiopia); Juliana Ferreira (University of São Paulo, São Paulo, Brasil); Gabriela Galli (University of Pittsburgh School of Medicine, Pittsburgh, PA); Tewodros Haile (Department of Internal Medicine, College of Health Sciences, Addis Ababa University); Bhakti Hansoti (Department of Emergency Medicine, Johns Hopkins University School of Medicine, Baltimore, MD); Madiha Hashmi (Ziauddin University, Karachi, Pakistan); Rebecca Inglis (Lao-Oxford-Mahosot Hospital-Wellcome Trust Research Unit [LOMWRU], Mahosot Hospital, Vientiane, Lao People's Democratic Republic and Department of Intensive Care, Oxford University Hospital Trust, Oxford, United Kingdom); Burton W. Lee (NIH, Bethesda, MD; University of Pittsburgh School of Medicine, Pittsburgh, PA); Faith Lelei (AIC Kijabe Hospital, Kijabe, Kenya); Ganbold Lundeg (Mongolian National University of Medical Sciences, Ulan Bator, Mongolia); David Misango (The Aga Khan University, Nairobi, Kenya); Ary Serpa Neto (Department of Critical Care Medicine and Institute of Education and Research, Hospital Israelita Albert Einstein, São Paulo, Brazil; Department of Intensive Care, Amsterdam University Medical Centers, location "AMC'", University of Amsterdam, Amsterdam, The Netherlands); Alfred Papali (Atrium Health, Charlotte, NC); Casey Park (Interdepartmental Division of Critical Care Medicine, Sunnybrook Health Sciences Centre, University of Toronto, Toronto, Canada); Rajyabardhan Pattnaik (Ispat General Hospital, Rourkela, India); Jennifer L. Pigoga (Division of Emergency Medicine, University of South Africa, Cape Town, South Africa); Luigi Pisani (Department of Anesthesia and Intensive Care, Miulli Regional Hospital, Acquaviva delle Fonti, Italy; Doctors with Africa - CUAMM, Padova, Italy; Mahidol-Oxford Tropical Medicine Research Unit (MORU), Mahidol University, Bangkok, Thailand); Elisabeth D. Riviello (Division of Pulmonary, Critical Care, and Sleep Medicine, Beth Israel Deaconess Medical Center and Harvard Medical School, Boston, MA); Kristina E. Rudd (University of Pittsburgh School of Medicine, Pittsburgh, PA); Marcus Schultz (Mahidol University, Bangkok, Thailand; University of Oxford, Oxford, United Kingdom; Amsterdam University Medical Centers, location "AMC," Amsterdam, The Netherlands); Varun U.
Shetty (University of Pittsburgh Medical Center, Pittsburgh, PA); Gentle S. Shrestha (Tribhuvan University Teaching Hospital, Kathmandu, Nepal); Chaisith Sivakorn (Mahidol University, Salaya, Thailand); Shaurya Taran (Interdepartmental Division of Critical Care Medicine, Sunnybrook Health Sciences Centre, University of Toronto, Toronto, Canada); T. Eoin West (University of Washington, Seattle, WA).

Group members of the subgroup "Triage": Lia M. Barros (Division of Cardiology, University of Washington Medical Center, Seattle, WA), Jennifer L. Pigoga (Division of Emergency Medicine, University of South Africa, Cape Town, South Africa), Sopheakmoniroth Chea (Department of Cardiology, Calmette Hospital, Cambodia), Bhakti Hansoti (Department of Emergency Medicine, Johns Hopkins University, Baltimore, MD), Sarah Hirner (School of Medicine, University of Colorado, Denver, Colorado), Alfred Papali (Division of Pulmonary and Critical Care Medicine, Atrium Health, Charlotte, NC), Kristina Rudd (Department of Critical Care Medicine, University of Pittsburgh, Pittsburgh, PA), Marcus Schultz (Mahidol University, Bangkok, Thailand; University of Oxford, Oxford, United Kingdom; Amsterdam University Medical Centers, location "AMC," Amsterdam, The Netherlands), and Emilie J. Calvello Hynes (Department of Emergency Medicine, University of Colorado, Denver, Colorado).

\section{REFERENCES}

1. Zhang J, Zhou L, Yang Y, Peng W, Wang W, Chen X, 2020. Therapeutic and triage strategies for 2019 novel coronavirus disease in fever clinics. Lancet Respir Med 8: e11-e12.

2. Wang $Q$, Wang $X$, Lin $H, 2020$. The role of triage in the prevention and control of COVID-19. Infect Control Hosp Epidemiol 41: 772-776.

3. Davarpanah AH et al., 2020. Novel screening and triage strategy in Iran during deadly coronavirus disease 2019 (COVID-19) epidemic: value of humanitarian teleconsultation service. J Am Coll Radiol 17: 734-738.

4. Chen S, Zhang Z, Yang J, Wang J, Zhai X, Bärnighausen T, Wang C, 2020. Fangcang shelter hospitals: a novel concept for responding to public health emergencies. Lancet 395: 13051314.

5. Erika $P$, Andrea V, Cillis MG, loannilli E, lannicelli $T$, Andrea $M$, Triage decision-making at the time of COVID-19 infection: the Piacenza strategy. Intern Emerg Med 15: 879-882.

6. Piliego C, Strumia A, Stone MB, Pascarella G, 2020. The ultrasound guided triage: a new tool for prehospital management of COVID-19 pandemic. Anesth Analg 131: e93-e94.

7. Carenzo L et al., 2020. Hospital surge capacity in a tertiary emergency referral centre during the COVID-19 outbreak in Italy. Anaesthesia 75: 928-934.

8. Zimmerman RK, Nowalk MP, Bear T, Taber R, Clarke KS, Sax TM, Eng H, Balasubramani GK, 2020. Proposed clinical indicators for efficient screening and testing for COVID-19 infection from classification and regression trees (CART) analysis. Hum Vaccin Immunother 1-4. doi: 2020.05.11.20097980.

9. Shi $Y$ et al., 2020. A quickly, effectively screening process of novel corona virus disease 2019 (COVID-19) in children in Shanghai, China. Ann Transl Med 8: 241.

10. Ma J, Shi X, Xu W, Lv F, Wu J, Pan Q, Yang J, Yu J, Cao H, Li L, 2020. Development and validation of a risk stratification model for screening suspected cases of COVID-19 in China. Aging 12: 13882-13894.

11. World Health Organization, 2020. Operational Considerations for Case Management of COVID-19 in Health Facility and Community Interim Guidance. Geneva, Switzerland: WHO.

12. World Health Organization, 2020. World Health Organization COVID-19: Case Definitions. Case Definitions Updated in Public Health Surveillance for COVID-19. Geneva, Switzerland: WHO.

13. World Health Organization, 2020. Clinical Management of COVID19 Interim Guidance. Geneva, Switzerland: WHO.

14. Rhee C, Baker M, Vaidya V, Tucker R, Resnick A, Morris CA, Klompas M; CDC Prevention Epicenters Program, 2020. Incidence of nosocomial COVID-19 in patients hospitalized at a large US academic medical center. JAMA Netw Open 3: e2020498.

15. World Health Organization, 2020. WHO COVID-19: Case Definitions. Geneva, Switzerland: WHO.

16. Guan W-j et al., 2020. Clinical characteristics of coronavirus disease 2019 in China. N Eng J Med 382: 1708-1720. 
17. Burke R, Killerby M, Newton S, Ashworth C, Berns A, Brennan S, 2020. Symptom profiles of a convenience sample of patients with COVID-19-United States, January-April 2020. Morb Mortal Wkly Rep 69: 904-908.

18. Smith B, Chu LK, Smith TC, Amoroso PJ, Boyko EJ, Hooper TI, Gackstetter GD, Ryan MAK; Millennium Cohort Study Team, 2008. Challenges of self-reported medical conditions and electronic medical records among members of a large military cohort. BMC Med Res Methodol 8: 37.

19. Zhang ZL, Hou YL, Li DT, Li FZ, 2020. Laboratory findings of COVID-19: a systematic review and meta-analysis. Scand $J$ Clin Lab Invest 80: 441-447.

20. Jain V, Yuan J-M, 2020. Predictive symptoms and comorbidities for severe COVID-19 and intensive care unit admission: a systematic review and meta-analysis. Int $J$ Pub Health 65: 533-546.

21. Sun $\mathrm{H}$ et al., 2020. COVID-19 outpatient screening: a prediction score for adverse events. medRxiv. doi: 2020.06.17.20134262.

22. Sun Q, Qiu H, Huang M, Yang Y, 2020. Lower mortality of COVID19 by early recognition and intervention: experience from Jiangsu province. Ann Intensive Care 10: 33.

23. Zhang $\mathrm{C}$ et al., 2020. A novel scoring system for prediction of disease severity in COVID-19. Front Cell Infect Microbiol 10: 318.

24. Dong $Y$ et al., 2020. A novel simple scoring model for predicting severity of patients with SARS-CoV-2 infection. Transbound Emerg Dis 67: 2823-2829.

25. Li Q, Zhang J, Ling Y, Li W, Zhang X, Lu H, Chen L, 2020. A simple algorithm helps early identification of SARS-CoV-2 infection patients with severe progression tendency. Infection 48: 577-584.

26. Gong $J$ et al., 2020. A tool for early prediction of severe coronavinus disease 2019 (COVID-19): a multicenter study using the risk nomogram in Wuhan and Guangdong, China. Clin Infect Dis 71: 833-840.

27. Zou X, Li S, Fang M, Hu M, Bian Y, Ling J, Yu S, Jing L, Li D, Huang $\mathrm{J}, 2020$. Acute physiology and chronic health evaluation II score as a predictor of hospital mortality in patients of coronavirus disease 2019. Crit Care Medicine 48: e657-e665.

28. Liang W et al.; China Medical Treatment Expert Group for COVID19, 2020. Development and validation of a clinical risk score to predict the occurrence of critical illness in hospitalized patients with COVID-19. JAMA Intern Med 180: 1081-1089.

29. Zhang $S$ et al., 2020. Development and validation of a risk factorbased system to predict short-term survival in adult hospitalized patients with COVID-19: a multicenter, retrospective, cohort study. Crit Care 24: 438

30. Xiao LS, Zhang WF, Gong MC, Zhang YP, Chen LY, Zhu HB, Hu CY, Kang P, Liu L, Zhu H, 2020. Development and validation of the HNC-LL score for predicting the severity of coronavirus disease 2019. EBioMedicine 57: 102880.

31. Tsui ELH, Lui C, Woo PPS, Cheung ATL, Lam PKW, Tang TW, Yiu CF, Wan CH, Lee LHY, 2020. Development of a data-driven COVID-19 prognostication tool to inform triage and step-down care for hospitalised patients in Hong Kong: a population based cohort study. medRxiv. doi: 2020.07.13.20152348.

32. Zhao L, Yu K, Zhao Q, Tian R, Xie H, Xie L, Deng P, Xie G, Bao A, Du $\mathrm{J}, 2020$. Lung ultrasound score in evaluating the severity of coronavirus disease 2019 (COVID-19) pneumonia. Ultrasound Med Biol 46: 2938-2944.

33. Peng $X$, Subbe CP, Zhang L, Luo Z, Peng L, 2020. NEWS can predict deterioration of patients with COVID-19. Resuscitation 152: 26-27.

34. Bi X, Su Z, Yan H, Du J, Wang J, Chen L, Peng M, Chen S, Shen B, $\mathrm{Li}$ J, 2020. Prediction of severe illness due to COVID-19 based on an analysis of initial fibrinogen to albumin ratio and platelet count. Platelets 31: 674-679.

35. Fan G et al., 2020. Comparison of severity scores for COVID-19 patients with pneumonia: a retrospective study. Eur Respir J 56: 2002113.

36. Hu H, Yao N, Qu Y, 2020. Comparing rapid scoring systems in mortality prediction of critically ill patients with novel coronavirus disease. Acad Emerg Med 27: 461-468.

37. Satici $C$ et al., 2020. Performance of pneumonia severity index and CURB-65 in predicting 30-day mortality in patients with COVID-19. Int J Infect Dis 98: 84-89.

38. Ufuk F, Demirci M, Sagtas E, Akbudak IH, Ugurlu E, Sari T, 2020. The prognostic value of pneumonia severity score and pectoralis muscle area on chest CT in adult COVID-19 patients. Eur J Radiol 131: 109271.

39. Reynolds TA, Bisanzo M, Dworkis D, Hansoti B, Obermeyer Z, Seidenberg P, Hauswald M, Mowafi H, 2013. Research priorities for data collection and management within global acute and emergency care systems. Acad Emerg Med 20: 1246-1250.

40. Starr N et al., 2020. Pulse oximetry in low-resource settings during the COVID-19 pandemic. Lancet Glob Health 8: e1121-e1122.

41. Wheeler I, Price C, Sitch A, Banda P, Kellett J, Nyirenda M, Rylance $\mathrm{J}, 2013$. Early warning scores generated in developed healthcare settings are not sufficient at predicting early mortality in Blantyre, Malawi: a prospective cohort study. PLoS One 8: e59830.

42. Ayebare RR, Flick R, Okware S, Bodo B, Lamorde M, 2020. Adoption of COVID-19 triage strategies for low-income settings. Lancet Respir Med 8: e22.

43. Long C, Xu H, Shen Q, Zhang X, Fan B, Wang C, Zeng B, Li Z, LiX, $\mathrm{Li} \mathrm{H}, 2020$. Diagnosis of the coronavirus disease (COVID-19): rRT-PCR or CT? Eur J Radiol 126: 108961.

44. Prokop M et al.; COVID-19 Standardized Reporting Working Group of the Dutch Radiological Society, 2020. CO-RADS - a categorical CT assessment scheme for patients with suspected COVID-19: definition and evaluation. Radiology 296: E97-E104.

45. Wong HYF et al., 2020. Frequency and distribution of chest radiographic findings in COVID-19 positive patients. Radiology 296: E72-E78.

46. American College of Radiology, 2020. ACR Recommendations for the Use of Chest Radiography and Computed Tomography (CT) for Suspected COVID-19 Infection. Reston, VA: American College of Radiology. Available at: https://www.acr.org/Advocacyand-Economics/ACR-Position-Statement/Recommendations-forChest-Radiography-and-CT-for-Suspected-COVID-19-Infection.

47. Poggiali E, Dacrema A, Bastoni D, Tinelli V, Demichele E, Mateo Ramos P, Marcianò T, Silva M, Vercelli A, Magnacavallo A, 2020. Can lung US help critical care clinicians in the early diagnosis of novel coronavirus (COVID-19) pneumonia? Radiology 295: E6.

48. Vetrugno L, Bove T, Orso D, Barbariol F, Bassi F, Boero E, Ferrari $\mathrm{G}$, Kong R, 2020. Our Italian experience using lung ultrasound for identification, grading and serial follow-up of severity of lung involvement for management of patients with COVID-19. Echocardiography 37: 625-627.

49. Soldati G et al., 2020. Proposal for international standardization of the use of lung ultrasound for patients with COVID-19. $J$ Ultrasound Med 39: 1413-1419.

50. Duca A, Piva S, Focà E, Latronico N, Rizzi M, 2020. Calculated decisions: Brescia-COVID Respiratory Severity Scale (BCRSS)/ algorithm. Emerg Med Pract 22 (Suppl 5): CD1-CD2.

51. Côté A, Ternacle J, Pibarot $P, 2020$. Early prediction of the risk of severe coronavirus disease 2019: a key step in therapeutic decision making. EBioMedicine 59: 102948.

52. Manivel V, Lesnewski A, Shamim S, Carbonatto G, Govindan T, 2020. CLUE: COVID-19 lung ultrasound in emergency department. Emerg Med Australas 32: 694-696.

53. Rubin GD et al., 2020. The role of chest imaging in patient management during the COVID-19 pandemic: a multinational consensus statement from the Fleischner society. Radiology 296: 172-180.

54. Hu L, Wang C, 2020. Radiological role in the detection, diagnosis and monitoring for the coronavirus disease 2019 (COVID-19). Eur Rev Med Pharmacol Sci 24: 4523-4528.

55. Henwood PC, Mackenzie DC, Rempell JS, Murray AF, Leo MM, Dean AJ, Liteplo AS, Noble VE, 2014. A practical guide to selfsustaining point-of-care ultrasound education programs in resource-limited settings. Ann Emerg Med 64: 277-285.e2.

\section{APPENDIX}

\section{Development of recommendations and suggestions.}

Selection of Task Force members. The selection of the group members was based on interest in specific aspects of COVID-19 and hands-on experience in triage, emergency rooms, high dependency unit (HDU) or intensive care units (ICUs) in LMICs. Alfred Papali and Marcus Schultz contacted potential team members through email early in the pandemic of COVID-19, and created eight subgroups assigned to separate areas in COVID-19 management: 'triage', 'safety', 'organization', 
'microbiology and laboratory tests, imaging tools, and diagnostic and prognostic modeling', 'acute respiratory failure, 'acute kidney injury', 'coagulopathy, 'prevention and therapy', 'shock' and 'support after initial care'. In total, there were 38 Task Force members representing five medical specialties or disciplines (emergency medicine, intensive care, infectious diseases, internal medicine and critical care nursing) from five out of six World Health Organization (WHO) geographic regions. The Task Force consisted of 16 full-time LMIC members, 16 full time high-income country (HIC) members-all with direct LMIC experience-and 6 members with joint LMIC/HIC appointments.

Selection of subgroup members. Lia M. Barros, Jennifer Pigoga, Alfred Papali, Bhakti Hansoti, Kristina E. Rudd, Sarah Hirner, Sopheakmoniroth Chea, Marcus Schultz, and Emilie Calvello Hynes were assigned to this subgroup based on their specific expertise and interest in triage and emergency medicine.

Meetings. Subgroup heads were initially contacted via email to establish the procedures for literature review and drafting of tables for evidence analysis. The subgroup heads continued work via the Internet. Several meetings occurred through teleconferences and electronic-based discussions among the subgroup heads and with members of other subgroups.

The subgroup head then drafted a live document to organize subgroup members, contacts, questions, and a working schedule for drafts. In the first electronic based discussion, a set of clearly defined questions were formulated regarding screening, triage, and prognostication, and use of diagnostic

TABLE A1

Quality of Evidence

\begin{tabular}{lll}
\hline A & Randomized clinical trials & High \\
B & $\begin{array}{c}\text { Downgraded randomized clinical trial(s) or } \\
\text { upgraded observational studies }\end{array}$ & Moderate \\
C & $\begin{array}{l}\text { Observational studies } \\
\text { D }\end{array}$ & Lowngraded observational studies or \\
& expert opinions & Very Low
\end{tabular}

Factors that may decrease strength of evidence: poor quality of planning and implementation of available RCTs, suggesting high likelihood of bias; inconsistency of results, including problems with subgroup analyses; indirectness of evidence (differing population, intervention, control, outcomes, comparison); imprecision of results; and high likelihood of reporting bias. Factors that may increase strength of evidence: large magnitude of effect (direct evidence, relative risk $>2$ with no plausible confounders); very large magnitude of effect with relative risk > 5 and no threats to validity (by two levels); and dose-response gradient. Evidence was then used to derive recommendations, which were ranged as strong or weak, putting emphasis on factors that play a strong role in whether or not these recommendations could be implemented in LMICS, such as safety, availability, and recomily. and weak recommendations as 'we suggest. ... A number of recommendations could remain 'ungraded' (UG), when, in the opinion of the subgroup members, such recommendations were not conducive for the process described above. The factors influencing these classifications are presented in Table A2. tools in relation to these topics. These were edited for content and clarity by the subgroup members. Following review of questions by the larger Task Force, Triage subgroup members conducted comprehensive reviews of the relevant literature to identify evidence for recommendations related to each of the posed questions. Each member of the triage subgroup summarized the evidence of their assigned question in an online supplement, and formulated a set of recommendations and suggestions after electronic-based discussions. After the approval of each subgroup member, the subgroup heads summarized the evidence in a report, which was sent for approval by all members of the other groups.

Search techniques. Literature searches for all questions were conducted through November 4, 2020 in four general medical databases (PubMed, Medline, Embase, and Web of Science), the Cochrane Libraries, and Google Scholar. Furthermore, subgroup members identified investigations from LMICs, and searched for yet unpublished study results. Specific search terminology is noted for each question in its respective section. Reference lists of all included articles were manually screened for additional pertinent literature. It is important to note that the COVID-19 literature base is expanding at an unprecedented pace. All studies through the time of writing were considered for inclusion in this review; however, some were excluded due consensus on their limited relevance to LMICs and others may be missing if released sometime after the review end date.

Articles were screened at the title, abstract, and full-text level to determine relevance to this review's questions. Those that provided information that may pose a solution to a study question were included.

Grading of Recommendations. In order to contextualize such data, we classified each piece of evidence as being of high or low quality, based largely on the design of the study that generated it; these classifications are defined in Appendix Table A1.

Reporting. The report was edited for style and form by Alfred Papali or Marcus Schultz, with final approval by subgroup heads and then by the entire COVID-LMIC Task Force. A final document was submitted to the 'American Journal of Tropical Medicine and Hygiene' for potential publication as an open access article.

Conflicts of interest. No members of the 'triage' subgroup represented industry, and there was no industry input into guidelines development. No member of the 'triage' subgroup received honoraria for any role in the guideline development process. None reported conflicts of interest. Open access fees for this manuscript, and all 9 others in the series, were supported by the Wellcome Trust of Great Britain.

TABLE A2

Strong vs. Weak Recommendations*

What is Considered

High or moderate evidence

Certainty about the balance of benefits

vs. harms and burdens

Certainty in or similar values

Resource implications

Availability and feasibility in LMICs Affordability for LMICs

Safety of the intervention in LMICs
How it effects the recommendation

The higher the quality of evidence, the more likely a strong recommendation.

The larger/smaller the difference between the desirable and undesirable consequences and the certainty around that difference, the more likely a strong/weak recommendation.

The more certainty or similarity in values and preferences, the more likely a strong recommendation.

The lower/higher the cost of an intervention compared to the alternative the more likely a strong/ weak recommendation.

The less available, the more likely a weak recommendation.

The less affordable, the more likely a weak recommendation.

The less safe in an LMIC, the more likely a weak recommendation. 